\title{
Frecuencia de riesgo neurobiológico en recién nacidos
}

\author{
LISSETH BARRA C. ${ }^{1}$, RUBÉN ALVARADO M. ${ }^{2}$ \\ 1. Kinesióloga, Magíster en Salud Pública. Escuela de Kinesiología, Pontificia Universidad Católica de Valparaíso, Valparaíso. \\ 2. Médico - Psiquiatra. Magíster en Salud Pública, PhD en Psiquiatría Comunitaria. Escuela de Salud Pública, \\ Facultad de Medicina, Universidad de Chile, Santiago.
}

\begin{abstract}
Neurobiological risk frequency on newborns

Introduction: High-risk or neurobiological vulnerable children are those who are more likely to have alterations in their psychomotor development during their pre-, peri-, or post-natal periods. Evidence suggests that these children must have early medical care. Objective: To describe the frequency of high-risk neurobiological infants born during 2008 in Viña del Mar Quillota public health hospitals. Methods: A descriptive design was used based on the analysis of existing information in the maternity and neonatology record books regarding live births in public hospitals of this service network. Results: The high-risk children accounted for $21.01 \%$ of all live births. The most frequent biological risk factors were prematurity $(11.4 \%)$, intrauterine growth retardation $(9.0 \%)$ and low birth weight $(7.8 \%)$. Conclusion: This epidemiological study represents the starting point for exploring the existing need to have statistical records of the various risk factors as well as have formal spaces for early treatment in the Quillota Viña del Mar health service to prevent and treat abnormal psychomotor development in all children with neurobiological risk.
\end{abstract}

(Key words: Neurobiological risk, psychomotor development, early medical care).

Rev Chil Pediatr 2012; 83 (6): 552-562

\section{RESUMEN}

Introducción: Los niños de alto riesgo o vulnerabilidad neurobiológica son aquellos que por sus antecedentes pre, peri o postnatales tienen una mayor probabilidad de presentar alteraciones en su desarrollo psicomotor. La evidencia sugiere que estos niños deben ingresar a Programas de Atención Temprana. Objetivo: Describir la frecuencia de niños de alto riesgo neurobiológico que nacieron en hospitales públicos del Servicio de Salud Viña del Mar Quillota, durante al año 2008. Paciente y Método: Se utilizó un diseño descriptivo a partir del análisis de información existente en los libros de registros de los servicios de maternidad y neonatología, del

Recibido el 03 de marzo de 2012, devuelto para corregir el 26 de marzo de 2012, segunda versión el 18 de abril de 2012 , tercera versión el 20 de junio de 2012, cuarta versión el 20 de junio de 2012 aceptado para publicación el 10 de agosto de 2012.

Este trabajo cumple con los requisitos sobre consentimiento /asentimiento informado, comité de ética, financiamiento, estudios animales y sobre la ausencia de conflictos de intereses según corresponda.

Correspondencia a:

Lisseth Barra Cabello

E-mail: lisseth.barra@ucv.cl 
universo de niños nacidos vivos en hospitales públicos de la red de este Servicio. Resultados: Los niños de alto riesgo representaron un $21,01 \%$ del total de recién nacidos vivos. Los factores de riesgo biológico más frecuentes fueron la prematurez $(11,4 \%)$, el retardo de crecimiento intrauterino $(9,0 \%)$ y el bajo peso de nacimiento $(7,8 \%)$. Conclusión: Este estudio epidemiológico, representa el punto de partida para explorar la necesidad existente de disponer de registros estadísticos de los distintos factores de riesgo biológico y contar con espacios formales para realizar atención temprana en el servicio de salud Viña del Mar Quillota con el propósito de prevenir y tratar alteraciones del desarrollo psicomotor en todos los niños con riesgo neurobiológico. (Palabras clave: Alto Riesgo Neurobiológico, Desarrollo Psicomotor, Atención Temprana).

Rev Chil Pediatr 2012; 83 (6): 552-562

\section{Introducción}

El Desarrollo Psicomotor (DSM) normal de un niño depende no sólo de la indemnidad del Sistema Nervioso, sino también de que este reciba estímulos adecuados en el momento adecuado $^{1}$. Por otra parte, la presencia de factores de riesgo biológico (FRB) puede perjudicar directamente este desarrollo o hacer a un niño más vulnerable ante estímulos medioambientales más adversos ${ }^{2-4}$.

Los niños que tienen FRB, tales como el retardo del crecimiento intrauterino, la prematurez, la hemorragia intracraneana y otras patologías, tienen más probabilidades de presentar problemas en su desarrollo psicomotor, ya sean cognitivos, motores, sensoriales o de comportamiento pudiendo ser estos, transitorios o definitivos. Por esta razón, los niños que presentan estos antecedentes se consideran de "alto riesgo" o "vulnerabilidad biológica" ${ }^{5-9}$.

La evidencia sugiere que estos niños debiesen ingresar a programas de atención tempra$\mathrm{na}^{4,10}$. La Atención Temprana se define como el conjunto de intervenciones dirigidas al niño, padres y entorno para favorecer el DSM. Es un modelo de abordaje dirigido a los niños que tienen riesgo de presentar alteraciones del DSM, o que ya presentan alteraciones del desarrollo que pueden ser transitorias y/o permanentes ${ }^{11}$. Entre las diversas referencias que hablan de la atención temprana, cabe destacar el "Libro Blanco de la Atención Temprana"" el cuál es un documento realizado por especialistas y asociaciones de la atención temprana de España. En dicho texto, al igual que en otras referencias, se describen los antecedentes para catalogar a un niño como de Alto Riesgo Bio- lógico (ARB), los cuáles debiesen ser utilizados como criterios de derivación a centros de atención temprana. Además, se señalan las estrategias en los distintos niveles de prevención de salud que deben realizarse en este tipo de programas $^{5-7,11}$.

\section{Atención temprana en Chile}

El concepto de Atención Temprana ha ido evolucionando hasta llegar a su definición actual. Inicialmente la "estimulación precoz", como se denominaba en aquel entonces, se dirigía a tratar a niños con patología establecida y tenía un carácter rehabilitador, lo que en el ámbito de la salud pública se conoce como nivel de prevención terciaria en salud ${ }^{6}$.

El modelo de rehabilitación infantil más conocido y de mayor trayectoria en nuestro país es Teletón, institución privada sin fines de lucro que se enfoca a tratar a niños con compromiso motor ocasionado por patologías neuro-músculo-esqueléticas ${ }^{12}$. Por consiguiente, esta institución representa un nivel de prevención terciario. En relación a otros trastornos de desarrollo (no motores) o para dar continuidad al trabajo de rehabilitación que se realiza en Teletón, existen diversas instituciones creadas por asociaciones de padres cuyos hijos presentan dichas patologías y/o profesionales de salud. Entre estas instituciones en la $\mathrm{V}$ región existe el centro Tukuipaj, ubicado en Villa Alemana, el cual es una organización no gubernamental que se preocupa por tratar a niños con discapacidad intelectual y motora. Para beneficio de los niños con trastornos del espectro autista, existe en Viña la Corporación Andalué. Para los niños con síndrome de Down existen distintas posibilidades en la 
V Región: APARID (Agrupación de Padres y Amigos por la Rehabilitación del niño con Síndrome de Down), Corporación Renacer, Sendas, entre otras instituciones. También existen escuelas de lenguaje, las cuáles tratan a niños con trastornos específicos en esta área. Todos los centros mencionados corresponden a un nivel de atención terciaria de salud. La derivación a estos centros no tiene organización en red con el servicio de salud, los niños llegan derivados por médicos tratantes informados o por la preocupación de los padres en buscar una alternativa de rehabilitación para sus hijos una vez confirmado el diagnóstico.

La Atención Temprana, como se entiende el concepto en la actualidad, actúa también a nivel primario y secundario de atención de salud $^{11}$. Esto se fundamenta en el concepto de Neuroplasticidad, es decir, la capacidad del Sistema Nervioso (SN) de realizar modificaciones funcionales y/o estructurales en las conexiones del circuito neuronal. La neuroplasticidad es dependiente de la edad y la estimulación que recibe el SN. La neuroplasticidad fundamenta los conceptos de períodos críticos $\mathrm{y}$ ventanas de oportunidad que justifican un enfoque anticipatorio frente al daño potencial del DSM ${ }^{10}$.

Por otra parte, el Sistema "Chile Crece Contigo" ha implementado salas de estimulación de DSM en centros de atención primaria de Salud, las cuales representan un primer acercamiento a un modelo de atención temprana. Sin embargo, esta política de infancia no tiene estrategias concretas de abordaje del DSM en nivel secundario y/o terciario en el sector público de atención en salud. ¿Qué iniciativas hay en Chile en este nivel? En la Región Metropolitana existen 2 iniciativas que se destacan: NINEAS (niños con necesidades especiales de atención de salud) en el Servicio Metropolitano Sur Oriente el cual es un equipo de profesionales que ofrece atención integral a niños y jóvenes, desde recién nacidos hasta los 19 años, que padecen una condición crónica que resulta en una limitación de su capacidad para desarrollar actividades de acuerdo a su edad. Otra experiencia nacional se encuentra en el Servicio de Salud Metropolitano Oriente donde existe el Instituto Nacional de Rehabili- tación Pedro Aguirre Cerda, el cual es el único centro asistencial estatal en Chile, destinado a proporcionar atención integral a personas con discapacidad física menores de 25 años. Este instituto también cuenta con un Programa dirigido a prematuros extremos del Servicio de Salud al que pertenece.

Por otra parte, varios países, cuentan con programas pediátricos de seguimiento dirigidos a los niños de ARB, especialmente para aquellos con antecedentes de prematurez. En Chile, se inició el seguimiento de niños prematuros extremos en el año 200013. En el Servicio de Salud Viña del Mar Quillota (SSVQ), estos niños además se benefician de un Programa de Atención Kinésica Temprana que desarrolla la Escuela de Kinesiología de la Pontificia Universidad Católica de Valparaíso. Este programa representa la única instancia de rehabilitación infantil existente en este servicio y trata a niños prematuros con riesgo, alteraciones transitorias y con compromiso del desarrollo establecido, tal como propone el modelo de atención temprana. Cabe destacar, que en el último informe de la comisión de nacional de seguimiento del niño prematuro reconoce lo siguiente: "La rehabilitación es una deuda que existe y a la cual debemos encontrar modelos de atención que den respuesta a esta demanda urgente" 14 . Los programas de atención temprana responden a esta necesidad, a la vez que también pueden beneficiar a niños que presenten otros FRB que pueden ocasionar alteraciones del DSM, distintos a la prematurez.

\section{Incidencia de niños de alto riesgo neurobiológico en el SSVQ}

La prematurez es, en la actualidad, la causa más frecuente de parálisis cerebral ${ }^{15}$. Este factor tiene una incidencia de aproximadamente $6,9 \%$ del total de partos en Chile (año 2004) ${ }^{16}$. En el caso de los prematuros extremos (menores de 32 semanas de edad gestacional y/o menores de 1500 grs) la incidencia fue de $0,99 \%$ de recién nacidos vivos (RNV) durante el período 2000-2004. En el SSVQ, en el año 2000, la incidencia de prematuros extremos o de seguimiento fue de $0,6 \%{ }^{14}$.

El retardo del crecimiento intrauterino (RCIU) se presenta entre un 3 y un $10 \%$ de 
los embarazos. Esto se refiere a aquellos RN cuyo peso de nacimiento se encuentra bajo el percentil 10 de la curva de crecimiento intrauterino, en otras palabras son Pequeños para la edad gestacional (PEG) ${ }^{17}$. Se produce por causas maternas, placentarias y/o fetales, las cuales derivan en una insuficiencia placentaria que se manifiesta en una hipoalimentación del feto e hipoxia crónica intrauterina ${ }^{14}$. El RCIU puede hacerse irreversible después del nacimiento y manifestarse en el bebé en un desarrollo psicomotriz deficiente y provocar secuelas neurológicas 9 .

Pese a ser un hallazgo frecuente, la hiperbilirrubinemia también se considera un FRB. La evidencia señala que cuando la cantidad de bilirrubina libre no conjugada sobrepasa la capacidad de unión de la albúmina, cruza la barrera hematoencefálica y cuando ésta, está dañada, complejos de bilirrubina unida a la albúmina también pasan libremente al cerebro ${ }^{18}$. Antiguamente, el kernictoris (nombre que se da a la encefalopatía causada por hiperbilirrubinemia) era la principal causa de parálisis cerebral infantil ${ }^{19}$. Estudios post-mortern encontraron que la bilirrubina producía daño a nivel de los núcleos encefálicos. Esto era especialmente frecuente en los casos de incompatibilidad $\mathrm{ABO}$ o factor $\mathrm{RH}$ entre la gestante y el bebé. La ictericia en la mayoría de los casos es benigna, pero por su potencial neurotoxicidad, debe ser monitoreada muy de cerca para identificar neonatos que pueden desarrollar hiperbilirrubinemia severa y alteraciones neurológicas inducidas por la bilirrubina. Aunque se suele considerar de mayor riesgo la hiperbilirrubinemia que alcanza valores superiores a $20 \mathrm{ml} / \mathrm{dl}$ de sangre y/o que como tratamiento requiere exanguinotransfusión, hoy en día se reconoce que la concentración exacta de bilirrubina asociada con la encefalopatía bilirrubínica en el neonato a término saludable es impredecible ${ }^{20}$. Otra secuela neurosensorial asociada a la hiperbilirrubinemia es la hipoacusia ${ }^{17}$. Cabe destacar que la Academia Americana de Pediatría ha señalado explícitamente que es necesario desarrollar futuras investigaciones epidemiológicas para establecer cuál es la incidencia del daño asociado a hiperbilirrubinemia ${ }^{21}$.

Cabe notar, que sólo se encontraron datos que describen la incidencia más reciente de la prematurez para el año 2009 , a nivel nacional en ese año hubo un 7,2\% de RN menores de 37 semanas, de estos un $1,17 \%$ correspondió a menores de 32 semanas (prematuros extremos) ${ }^{22}$. No se encontraron referencias actuales nacionales y/o de este servicio para otros FRB como los ya mencionados. Por consiguiente, se desconoce qué porcentaje de los niños nacidos en el SSVQ debiesen ingresar a PAT y/o rehabilitación infantil en el SSVQ. En base a estos antecedentes, se observa la necesidad de contar con estudios epidemiológicos que aporten datos respecto a la magnitud de los FRB. Esto permitirá planificar acciones dirigidas al DSM en todos los niveles de atención de salud. El objetivo de este estudio fue describir la frecuencia de niños de $\mathrm{ARB}$, que nacieron en hospitales públicos de la red del SSVQ, durante el año 2008 e identificar los FRB más frecuentes en esta población.

\section{Pacientes y Método}

Se realizó un estudio descriptivo, basado en el análisis de información secundaria, del universo de niños nacidos en hospitales públicos de la red del SSVQ durante el año 2008. Se incluyó a los Recién Nacidos Vivos (RNV) con registros de su nacimiento hasta su egreso de la maternidad y/o neonatología. Se excluyeron los RNV cuyo lugar de residencia era fuera de la zona geográfica que corresponde a la red del SSVQ.

Los datos se recolectaron a partir de los registros de partos de las maternidades y libros de ingreso a neonatologías del SSVQ. Del total de partos, se seleccionó a los RNV que cumplían con los criterios ya señalados. Se realizó el análisis exploratorio completando los registros faltantes y corrigiéndolos en la medida de lo posible. Posteriormente, se usó el programa Stata 8 realizando estadística descriptiva. Para efectos del análisis de frecuencias de FRB y niños de $\mathrm{ARB}$, estos hospitales se dividieron en hospitales de menor y mayor complejidad, siendo estos últimos aquellos que cuentan con servicio de neonatología. Cabe señalar que para cuantificar los niños con vulnerabilidad biológica, que son los que debiesen ingresar 
a un programa de atención temprana, no se consideró a los niños que fallecieron en etapa neonatal.

Para medir la frecuencia de FRB se consideraron las siguientes variables: Prematurez, bajo peso al nacer (BPN), PEG, asfixia perinatal, hiperbilirrubinemia, convulsiones neonatales, lesión cerebral, genopatías y malformaciones del sistema nervioso. En el caso de la prematurez y el BPN se establecieron las categorías habitualmente descritas en la bibliografía ${ }^{16,17,23}$. Para el resto de las variables se evaluó si el RNV presentaba o no el FRB en estudio según lo señalado por los registros $\mathrm{y}$, en el caso de las genopatías, se registró su tipo.

Para medir la cantidad de niños de ARB, se consideró la variable: Incidencia de vulnerabilidad biológica, definida como total de niños que presentan al menos $1 \mathrm{FRB}$, entre los nacidos en el año 2008 en el SSVQ. Además, dado que se sabe que a mayor cantidad de FRB que presente un niño se incrementa el riesgo de presentar alteraciones del $\mathrm{DSM}^{5}$, se establecieron las siguientes categorías de vulnerabilidad: Sin Vulnerabilidad (RNV sin FRB), Menor Vulnerabilidad (RNV que presentan 1 FRB), Mediana Vulnerabilidad (RNV que presentan 2 FRB, a excepción de RNP con menos de $2500 \mathrm{~g}$ al nacer, pero que fueron AEG) y Alta Vulnerabilidad (RNV que presentan 3 o más FRB, son RNP extremos y/o presentan cromosomopatías o malformaciones del sistema nervioso). Tanto la incidencia total de vulnerabilidad como para las categorías señaladas fueron descritas en porcentaje.

A fin de resguardar los aspectos éticos, se solicitó las autorizaciones necesarias para acceder a los libros de registro de las maternidades y neonatologías del SSVQ y se adquirió el compromiso de que los datos de identificación personal, tales como el RUT y nombre, sólo se utilizarían para verificar la información y evitar un registro duplicado de casos, especialmente en los niños que son derivados desde las maternidades a las unidades de neonatología. Dado el objetivo y diseño del estudio, no se tomó contacto con padres, ni pacientes. Los datos solicitados sólo se usaron para fines de la investigación, resguardando la confidencialidad de los mismos.

\section{Resultados}

\section{Descripción general de la población en estudio}

Se identificó a 6796 RNV los cuales correspondieron a la población en estudio. De estos, el 51,0\% correspondió a RNV de sexo masculino. La edad gestacional promedio fue de 38,5 semanas, siendo el promedio más bajo el correspondiente al Hospital de Viña (38 semanas). En relación al peso de nacimiento, este fue en promedio de $3312,76 \mathrm{~g}$ para el total de los RNV. Los promedios de peso más bajo se presentaron en el hospital de Petorca y en el hospital de Viña. En relación a la talla promedio de nacimiento, esta fue de 49,5 centímetros, siendo también en este caso, el hospital de Viña quien presentó el promedio más bajo. Este dato que fue el que faltó con mayor frecuencia, donde un $8,6 \%$ de los RNV no tenía registrado este dato en el libro de partos. Sin embargo, dado que esta variable no se relaciona con los FRB estudiados, la ausencia de este dato no afecta los resultados del estudio (tabla $1)$.

\section{Frecuencia de vulnerabilidad o alto riesgo neurobiológico en $R \mathbf{N V}$}

En la población en estudio, un 78,5\% de los niños no presentó FRB. Los RNV con vulnerabilidad biológica representaron un total de $21,0 \%$, siendo la categoría de menor vulnerabilidad la más frecuente (figura 1). Los niños sin vulnerabilidad, nacieron en su mayoría en hospitales de menor complejidad. En cambio, los niños con vulnerabilidad neurobiológica nacieron principalmente en hospitales más complejos. Además, los niños de mayor vulnerabilidad, en su amplia mayoría nacieron en el Hospital de Viña del Mar (tabla 2).

\section{Frecuencia de FRB en RNV del SSVQ}

Los FRB más frecuentes fueron la prematurez $(11,4 \%)$, el retardo de crecimiento intrauterino $(9,0 \%)$ y el BPN $(7,9 \%)$. Al observar las subcategorías de estas variables se observa que los prematuros no extremos y los RNV menores de $2500 \mathrm{~g}$ fueron los casos más frecuentes con un $9,8 \%$ y $6,5 \%$ respectivamente (tabla $3)$. Los FRB menos frecuentes fueron las con- 


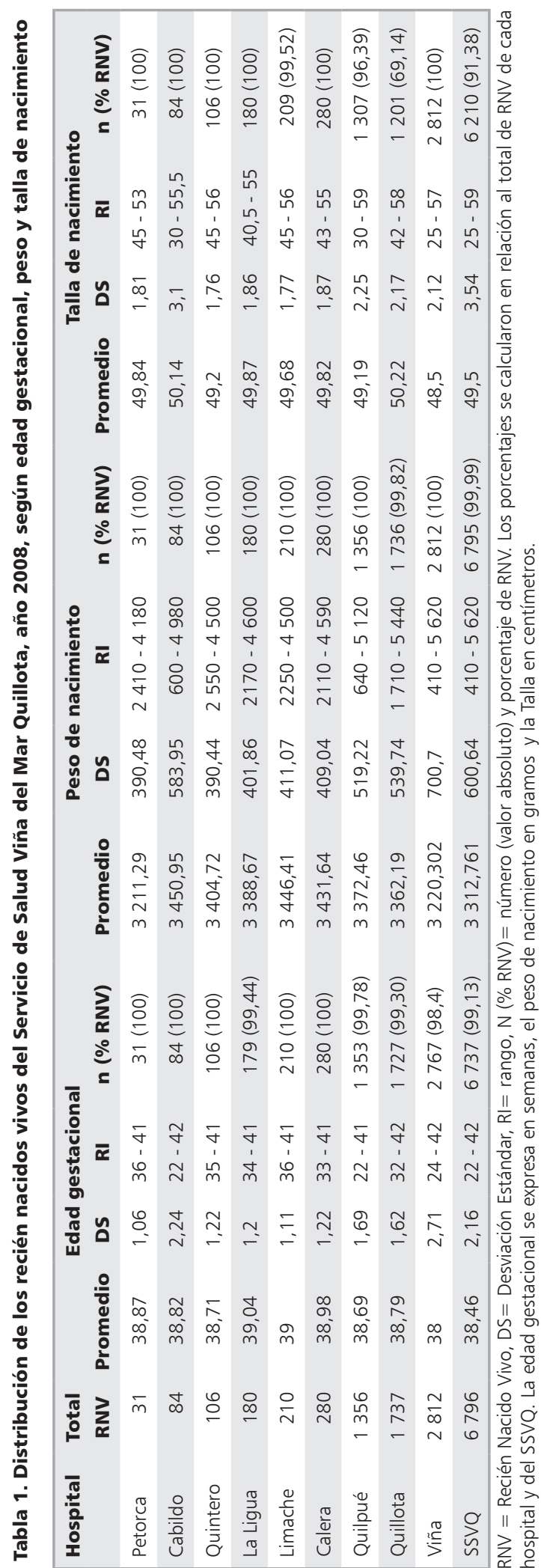

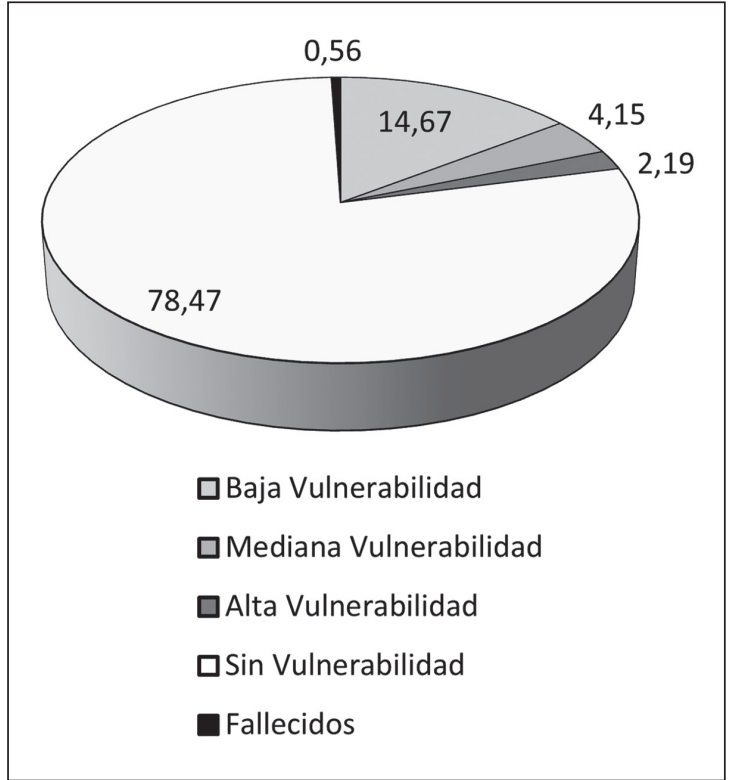

Figura 1. Distribución de frecuencia (en porcentaje) de los Recién Nacidos Vivos del Servicio de Salud Viña del Mar Quillota, según nivel de vulnerabilidad biológica, año 2008. *El porcentaje se calculó en relación a los $6796 \mathrm{RNV}$ año 2008 en SSVQ. Descripción de categorías: Sin Vulnerabilidad (RNV sin FRB), Menor Vulnerabilidad (RNV que presentan 1 FRB), Mediana Vulnerabilidad (RNV que presentan 2 FRB, a excepción de RNP con menos de $2500 \mathrm{~g}$ al nacer, pero que fueron AEG), Alta Vulnerabilidad (RNV que presentan 3 o más FRB, son RNP extremos y/o presentan cromosomopatías o malformaciones del sistema nervioso).

vulsiones y las malformaciones del $\mathrm{SN}$, ambas con un $0,1 \%$ de frecuencia. En el caso de las genopatías la más frecuente fue el síndrome de Down encontrándose 7 casos.

En los hospitales de menor complejidad los FRB más frecuentes en estos hospitales fueron: PEG $(0,7 \%)$, prematurez $(0,25 \%)$ y el BPN $(0,11 \%)$ Por otra parte, en los hospitales de mayor complejidad (aquellos que cuentan con servicio de neonatología), el FRB más frecuente fue la prematurez $(11,15 \%)$. Se observa, además que los prematuros extremos nacieron casi en su totalidad, en el Hospital de Viña donde hubo 100 de los 107 casos encontrados para todo el SSVQ. En el caso de los niños PEG, la frecuencia en estos hospitales fue de un $8,3 \%$. El tercer FRB más frecuente fue el BPN, que se presentó en un 7,8\% (tablas 4 y 5). 
Tabla 2. Distribución de frecuencia de los recién nacidos vivos del Servicio de Salud Viña del Mar Quillota, según hospital de nacimiento y vulnerabilidad biológica, año 2008

\begin{tabular}{|c|c|c|c|c|c|c|c|c|c|c|c|c|}
\hline \multirow[t]{3}{*}{ SSVQ } & \multicolumn{2}{|c|}{ Sin vulnerabilidad } & \multicolumn{8}{|c|}{ Con vulnerabilidad } & \multicolumn{2}{|c|}{ Fallecidos } \\
\hline & \multirow[b]{2}{*}{$\mathbf{n}$} & \multirow[b]{2}{*}{$\%$} & \multicolumn{2}{|c|}{ Baja } & \multicolumn{2}{|c|}{ Mediana } & \multicolumn{2}{|c|}{ Alta } & \multicolumn{2}{|c|}{ Total } & \multirow[b]{2}{*}{$\mathbf{n}$} & \multirow[b]{2}{*}{$\%$} \\
\hline & & & $\mathbf{n}$ & $\%$ & $\mathbf{n}$ & $\%$ & $\mathbf{n}$ & $\%$ & $\mathbf{n}$ & $\%$ & & \\
\hline Petorca & 26 & 83,87 & 4 & 12,9 & 1 & 3,226 & 0 & 0 & 5 & 16,13 & 0 & 0 \\
\hline Cabildo & 77 & 91,67 & 5 & 5,96 & 1 & 1,19 & 0 & 0 & 6 & 7,14 & 1 & 1,19 \\
\hline Quintero & 94 & 88,68 & 11 & 10,38 & 0 & 0 & 1 & 0,94 & 12 & 11,32 & 0 & 0 \\
\hline Limache & 209 & 99,52 & 0 & 0 & 1 & 0,5 & 0 & 0 & 1 & 0,48 & 0 & 0 \\
\hline La Ligua & 163 & 90,56 & 13 & 7,22 & 3 & 1,67 & 1 & 0,55 & 17 & 9,44 & 0 & 0 \\
\hline Calera & 262 & 93,57 & 15 & 5,36 & 1 & 0,36 & 2 & 0,71 & 18 & 6,43 & 0 & 0 \\
\hline Quilpué & 1053 & 77,65 & 241 & 17,77 & 47 & 3,47 & 15 & 1,1 & 303 & 22,34 & 3 & 0,22 \\
\hline Quillota & 1389 & 79,97 & 258 & 14,85 & 78 & 4,49 & 8 & 0,46 & 344 & 19,8 & 4 & 0,23 \\
\hline Viña & 2060 & 73,26 & 450 & 16 & 150 & 5,33 & 122 & 4,34 & 722 & 25,68 & 30 & 1,07 \\
\hline Total & 5333 & 78,47 & 997 & 14,67 & 282 & 4,15 & 149 & 2,19 & 1428 & 21,01 & 38 & 0,56 \\
\hline
\end{tabular}

*El porcentaje se calculó en relación a los 6796 RNV año 2008 en SSVQ.

SSVQ: Servicio de Salud Viña del Mar Quillota.

Tabla 3. Distribución de frecuencias y porcentajes de factores de riesgo biológico en recién nacidos vivos del Servicio de Salud Viña del Mar Quillota, durante el año 2008

\begin{tabular}{|c|c|c|}
\hline \multirow[t]{2}{*}{ Factores de Riesgo } & \multicolumn{2}{|c|}{ Total* } \\
\hline & $\mathbf{n}$ & $\%$ \\
\hline RNP & 775 & 11,4 \\
\hline No extremo & 668 & 9,83 \\
\hline Extremos & 107 & 1,57 \\
\hline BPN & 541 & 7,96 \\
\hline Bajo peso & 442 & 6,5 \\
\hline Muy bajo peso & 63 & 0,93 \\
\hline Extremo bajo peso & 36 & 0,53 \\
\hline PEG & 614 & 9,03 \\
\hline Asfixia perinatal & 105 & 1,55 \\
\hline Hiperbilirrubinemia & 320 & 4,71 \\
\hline Convulsiones neonatales & 8 & 0,12 \\
\hline Lesión cerebral & 20 & 0,29 \\
\hline Genopatías & 24 & 0,35 \\
\hline Malformaciones Congénitas SN & 8 & 0,12 \\
\hline
\end{tabular}

*El porcentaje se calculó en relación a los 6796 RNV año 2008 en SSVQ. RNP= Recién nacido prematuro, BPN= Bajo peso de nacimiento, $\mathrm{PEG}=$ Pequeño para la edad gestacional, $\mathrm{SN}=$ Sistema nervioso.

\section{Discusión}

En este estudio, la población estudiada correspondió a los RNV cuyo parto se produjo en hospitales pertenecientes a la red pública del SSQV. Un 21,01\% correspondió a casos de pacientes de alto riesgo neurobiológico. Sin embargo, es probable que al excluir RNV cuyo parto se produjo fuera de la red pública del SSVQ, también se excluyeron casos de niños pertenecientes a este servicio. Cabe señalar que no se incluyeron otros FRB que podrían ser importantes, tales como el consumo de drogas por parte de la madre durante la gestación, ya que este antecedente no suele registrarse en los libros de partos de las maternidades.

Por otro lado, aunque de los 3 servicios de salud existentes en la V región, el SSVQ, es el que tiene el mayor número de RNV, los resultados de este estudio no pueden extrapolarse en forma directa a nivel regional ni nacional. Además, es importante señalar que al incluir sólo los RNV del año 2008, no es posible establecer si los resultados representan una tendencia, y por lo tanto, inferir los resultados a otros años. Sin embargo, se sabe que en el caso de la prematurez, que es unos de los FRB 
Tabla 4. Frecuencia de factores de riesgo biológico en recién nacidos vivos del Servicio de Salud Viña del Mar Quillota, año 2008, en maternidades de hospitales de menor complejidad

\begin{tabular}{|c|c|c|c|c|c|c|c|c|c|c|c|c|c|c|}
\hline \multirow[t]{2}{*}{ Factores de riesgo } & \multicolumn{2}{|c|}{ Petorca } & \multicolumn{2}{|c|}{ Cabildo } & \multicolumn{2}{|c|}{ Quintero } & \multicolumn{2}{|c|}{ La Ligua } & \multicolumn{2}{|c|}{ Limache } & \multicolumn{2}{|c|}{ Calera } & \multicolumn{2}{|c|}{ Total } \\
\hline & $\mathbf{n}$ & $\%$ & $\mathbf{n}$ & $\%$ & $\mathbf{n}$ & $\%$ & $\mathbf{n}$ & $\%$ & $\mathbf{n}$ & $\%$ & $\mathbf{n}$ & $\%$ & $\mathbf{n}$ & $\%$ \\
\hline RNP & 1 & 3,23 & 4 & 4,76 & 4 & 3,77 & 2 & 1,11 & 4 & 1,9 & 2 & 0,71 & 17 & 0,25 \\
\hline No extremo & 1 & 3,23 & 3 & 3,57 & 4 & 3,77 & 2 & 1,11 & 4 & 1,9 & 2 & 0,71 & 16 & 0,24 \\
\hline Extremos & 0 & 0 & 1 & 1,19 & 0 & 0 & 0 & 0 & 0 & 0 & 0 & 0 & 1 & 0,01 \\
\hline BPN & 1 & 3,23 & 2 & 2,38 & 0 & 0 & 2 & 1,11 & 1 & 0,48 & 2 & 0,71 & 8 & 0,11 \\
\hline Bajo peso & 1 & 3,23 & 1 & 1,19 & 0 & 0 & 2 & 1,11 & 1 & 0,48 & 2 & 0,71 & 7 & 0,1 \\
\hline Muy bajo peso & 0 & 0 & 0 & 0 & 0 & 0 & 0 & 0 & 0 & 0 & 0 & 0 & 0 & 0 \\
\hline Extremo bajo peso & 0 & 0 & 1 & 1,19 & 0 & 0 & 0 & 0 & 0 & 0 & 0 & 0 & 1 & 0,01 \\
\hline PEG & 4 & 12,9 & 6 & 7,14 & 5 & 4,72 & 14 & 7,78 & 8 & 3,81 & 10 & 3,57 & 47 & 0,69 \\
\hline Asfixia perinatal & 0 & 0 & 1 & 1,19 & 2 & 1,89 & 1 & 0,56 & 1 & 0,48 & 5 & 1,79 & 10 & 0,15 \\
\hline Hiperbilirrubinemia & 0 & 0 & 0 & 0 & 0 & 0 & 0 & 0 & 0 & 0 & 0 & 0 & 0 & 0 \\
\hline Convulsiones neonatales & 0 & 0 & 0 & 0 & 0 & 0 & 1 & 0,56 & 0 & 0 & 0 & 0 & 1 & 0,01 \\
\hline Lesión cerebral & 0 & 0 & 0 & 0 & 0 & 0 & 0 & 0 & 0 & 0 & 0 & 0 & 0 & 0 \\
\hline Genopatías & 0 & 0 & 0 & 0 & 1 & 0,94 & 0 & 0 & 0 & 0 & 2 & 0,72 & 3 & 0,04 \\
\hline Malformaciones congénitas SN & 0 & 0 & 0 & 0 & 0 & 0 & 1 & 0,55 & 0 & 0 & 0 & 0 & 1 & 0,01 \\
\hline
\end{tabular}

*El porcentaje se calculó en relación a los 6796 RNV año 2008 en SSVQ. RNP= Recién nacido prematuro, BPN= Bajo peso de nacimiento, $\mathrm{PEG}=$ Pequeño para la edad gestacional, $\mathrm{SN}=$ Sistema nervioso.

Tabla 5. Frecuencia de factores de riesgo biológico en recién nacidos vivos del Servicio de Salud Viña del Mar Quillota, año 2008, en maternidades de hospitales de mayor complejidad

\begin{tabular}{|c|c|c|c|c|c|c|c|c|}
\hline \multirow[t]{2}{*}{ Factores de riesgo } & \multicolumn{2}{|c|}{ Quilpué } & \multicolumn{2}{|c|}{ Quillota } & \multicolumn{2}{|c|}{ Fricke } & \multicolumn{2}{|c|}{ Total * } \\
\hline & $\mathbf{n}$ & $\%$ & $\mathbf{n}$ & $\%$ & $\mathbf{n}$ & $\%$ & $\mathbf{n}$ & $\%$ \\
\hline RNP & 116 & 8,55 & 157 & 9,09 & 485 & 17,53 & 758 & 11,15 \\
\hline No extremo & 110 & 8,11 & 157 & 0,09 & 385 & 13,91 & 652 & 9,59 \\
\hline Extremos & 6 & 0,44 & 0 & 0 & 100 & 3,61 & 106 & 1,56 \\
\hline BPN & 61 & 4,49 & 106 & 6,14 & 366 & 13,02 & 533 & 7,84 \\
\hline Bajo peso & 57 & 4,2 & 106 & 6,14 & 272 & 9,67 & 435 & 6,4 \\
\hline Muy bajo peso & 3 & 0,22 & 0 & 0 & 60 & 2,13 & 63 & 0,93 \\
\hline Extremo bajo peso & 1 & 0,07 & 0 & 0 & 34 & 1,21 & 35 & 0,51 \\
\hline PEG & 138 & 10,2 & 166 & 9,55 & 263 & 9,35 & 567 & 8,34 \\
\hline Asfixia perinatal & 14 & 0,88 & 22 & 1,27 & 59 & 2,09 & 95 & 1,4 \\
\hline Hiperbilirrubinemia & 108 & 7,96 & 53 & 3,07 & 159 & 5,65 & 320 & 4,7 \\
\hline Convulsiones neonatales & 1 & 0,07 & 2 & 0,12 & 4 & 0,14 & 7 & 0,1 \\
\hline Lesión cerebral & 1 & 0,07 & 2 & 0,12 & 17 & 0,6 & 20 & 0,3 \\
\hline Genopatías & 3 & 0,22 & 5 & 0,29 & 13 & 0,46 & 21 & 0,31 \\
\hline Malformaciones congénitas SN & 3 & 0,22 & 1 & 0,06 & 3 & 0,11 & 7 & 0,1 \\
\hline
\end{tabular}

*El porcentaje se calculó en relación a los 6796 RNV año 2008 en SSVQ. RNP= Recién nacido prematuro, BPN= Bajo peso de nacimiento, $\mathrm{PEG}=$ Pequeño para la edad gestacional, $\mathrm{SN}=$ Sistema nervioso. 
más estudiados, existe una tendencia creciente tanto de la incidencia como de la sobreviven$\operatorname{cia}^{14,15,24-26}$.

Según los resultados de este estudio el FRB más frecuente fue la prematurez no extrema. La cifra es más alta que la reportada para igual período a nivel nacional y regional ${ }^{22}$. Esto pudiera deberse a que la población en estudio no consideró a los RNV de hospitales públicos de la región pertenecientes a otros servicios de salud, ni los RNV correspondientes a clínicas privadas. De hecho, el total de RNV considerados en este estudio representa un $28,63 \%$ de los RNV reportados para la región el año $2008^{27}$. Por otra parte, se debe tener presente que en este trabajo se consideró la actual definición de la OMS, que señala que un RNP es aquel cuya EG al momento del parto es menor de 37 semanas. La Academia Americana de Pediatría propone considerar prematuros a aquellos que nacen con menos de 38 semanas $^{28}$. Esto quiere decir, que si se hubiese utilizado este último criterio, la incidencia de la prematurez como FRB sería aún mayor, ya que de $11,4 \%$ hubiese aumentado a 20,39\%.

Es importante señalar que en la práctica clínica, muchos prematuros no extremos no requieren hospitalizaciones en neonatología, siendo probable que se olvide que son pacientes de riesgo neurobiológico. Las alteraciones del DSM en estos casos suelen detectarse en etapa escolar. La razón de esto, pudiera explicarse por lo que señala la Academia Americana de Pediatría y otros autores que incluso proponen llamar a estos niños prematuros tardíos ("Late preterm") y no "casi a término" como suelen llamarles. Estos autores señalan que la frecuencia y las secuelas que presentan estos niños han sido menos estudiadas que los prematuros extremos ${ }^{29}$.

Al analizar los resultados, también se observó que los hospitales de baja complejidad presentaron una frecuencia menor de casos de niños con FRB. Los prematuros extremos y/o recién nacidos con extremo bajo peso nacieron con mayor frecuencia en el hospital de Viña, que corresponde al hospital de mayor complejidad del SSVQ. Este hospital además, presentó los promedios de EG y talla más bajos. Esto se debe a que los embarazos considerados de alto riesgo obstétrico son derivados antes de la ocurrencia del parto a hospitales de mayor complejidad.

Además, es importante señalar que si un $\mathrm{RN}$ no requirió ser derivado a una unidad de cuidado intensivo neonatal, esto no implica necesariamente ausencia de vulnerabilidad neurobiológica $^{30}$. En este estudio, hubo casos de RNV sanos, es decir, que no presentaron ninguna patología, pero que presentaron FRB tales como los prematuros tardíos y/o PEG, los cuales aunque su vulnerabilidad es menor, aún tienen probabilidad de presentar alteraciones en su desarrollo. Recordemos que los trastornos de DSM pueden tener diversas manifestaciones y algunas suelen no pesquisarse en los primeros años sino en etapa escolar. Por tal motivo se sugiere que el seguimiento de niños de riesgo neurológico se realice a hasta dicha etapa.

En el caso del Programa de Atención Temprana a Prematuros de Seguimiento que se realiza en el Servicio de Salud Viña del Mar Quillota que es el servicio de salud donde se realizó este estudio, en el año 2008 se realizaron 124 ingresos, los cuales superan a los 106 casos de niños prematuros extremos que hubo en el SSVQ en aquel mismo año, debido a que también se ingresaron algunos casos de prematuros no extremos derivados desde el policlínico de neonatología donde se controlan. Sin embargo, estos 124 niños sólo representan un 8,7\% de los niños de ARB detectados en este estudio. No es posible conocer si el resto de los niños de ARB recibió atenciones en salas de estimulación del programa Chile Crece Contigo, porque no existen registros para constatarlo. Por lo demás, el énfasis de este programa está en la protección social ${ }^{31,32}$. Por otra parte, si bien existen centros de rehabilitación infantil, los cuáles no pertenecen a la red pública de salud, la atención de estas instituciones se dirige principalmente a niños que tienen un trastorno del desarrollo diagnosticado y no necesariamente en riesgo y/o con alteraciones transitorias del DSM.

Por consiguiente, en la actualidad, no es posible señalar con certeza cuál es la brecha existente entre los niños que presentan ARB y aquellos que tienen la posibilidad de ingresar a programas de atención temprana. Es necesario 
continuar estudiando la vulnerabilidad biológica de los RNV, a fin de tener cifras más precisas de lo que ocurre en la realidad nacional y tendencias a lo largo del tiempo.

En conclusión, este estudio epidemiológico aborda la necesidad de disponer de registros estadísticos de los FRB, con objetivo de identificar a los niños de alto riesgo neurológico que requieren intervención en programas de atención temprana para prevenir y tratar oportunamente las alteraciones del DSM.

\section{Agradecimientos}

Se agradece a los todos profesionales del SSVQ que autorizaron y facilitaron la labor de recolección de datos necesarios para la realización de este estudio, en especial a Maritza Cofré quien trabaja para la Dirección del Servicio.

\section{Referencias}

1.- Avaria M: Aspectos biológicos del desarrollo psicomotor. Rev Ped Elec [en línea] 2005. 2 (1).

2.- Chávez R: Neurodesarrollo Neonatal e Infantil: Un enfoque Multi-Inter y Transdisciplinario en la Prevención del Daño. Editorial Panamericana, 2003. Capítulo 19.

3.- Andraca I, Pino P, La Parra A, Rivera F, Castillo M: Factores de riesgo para el desarrollo psicomotor en niños nacidos en óptimas condiciones biológicas. Rev Saúde Publica 1998; 32 (2): 138-47.

4.- Engle P, Black M, Behrman J,et al and the International Child Development Steering Group: Strategies to avoid the loss of developmental potential in more than 200 million children in the developing world. Lancet 2007; 369: 229-42.

5.- Ramos I: Detección y Diagnóstico Precoz de los Trastornos del Desarrollo Psicomotor. VoxPaed 2007; 15 (1): 36-43.

6.- Botana Del Arco I: La Atención Temprana. IV Foro de Pediatría de Atención Primaria de Extremadura. Foro Pediátrico 2005; 2 (Abril, Supl 1): 32-7.

7.- Ramos I, Márquez A: Recién Nacido de Riesgo Neurológico. VoxPaed 2000; 8 (2): 5-10.

8.- Robles MC, Poo P, Olivé MLP: Atención Temprana: Recursos y Pautas de Intervención en Niños de Riesgo con Retrasos del Desarrollo. Protocolos de Neurología,
Asociación Española de Pediatría 2008; 37: 278-85.

9.- Bos A, Einspieler C, Prechtl H: Intrauterine growth retardation, general movements, and neurodevelopmental outcome: a review. Dev Med and Child Neurol 2001; 43: 61-8.

10.- Artigas J: Atención Precoz de los Trastornos del Neurodesarrollo. A favor de la Intervención Precoz de los Trastornos del Neurodesarrollo. Rev Neurol 2007; 44 (supl 3): s31-s34.

11.- GAT (Grupo de Atención Temprana): Libro Blanco de la Atención Temprana. Ed. Real Patronato de Prevención y Atención a personas con Minusvalía, 2000. Documentos 55/2000. Madrid Año 2000.

12.- Fundación Teletón \& Sociedad Pro Ayuda del Niño Lisiado, Memoria Anual 2009-2010.

13.- Morgues M, Henríquez MT, Tohá D, et al: Situación del Prematuro Menor de 1500 g en Chile. Comisión Nacional de Seguimiento del Prematuro. Minsal, 2001.

14.- Minsal: Análisis Epidemiológico de los Recién Nacidos Menores de 32 Semanas en la Red Pública de Salud de Chile. Quinquenio 2000-2004. Informe técnico. 2006.

15.- Pallás $C$ : Seguimiento neurológico del niño prematuro. III Jornada de Actualización en Pediatría de la Sociedad de Pediatría de Atención Primaria de Extremadura. Foro pediátrico 2004; 15: 4-10.

16.- Tapia JL, Ventura-Junca P: Manual de Neonatología. Editorial Mediterráneo, 2001.

17.- Tapia JL, González A: Neonatología. Editorial Mediterráneo, 2008. Tercera Edición.

18.- Ventura-Juncá P: Ictericia No Hemolítica del Recién Nacido de Término. En Manual de Pediatría de la Pontificia Universidad Católica de Chile. Disponible en: http://escuela.med.puc.cl/publ/ManualPed/RNIctericia. html, acceso el 29-05-2010.

19.- Muzaber L, Shapira I: Parálisis Cerebral y el Concepto Bobath de Neurodesarrollo. Rev Hosp Mat Inf Ramón Sardá 1998; 17 (2): 84-90.

20.- Mazzi E, et al: Actualización: Hiperbilirrubinemia Neonatal. Rev Soc Bol Ped 2005; 44 (1): 26-35.

21.- AAP (American Academy of Pediatrics): Clinical Practice Guideline: Management of Hyperbilirubinemia in the Newborn Infant 35 or More Weeks of Gestation. Pediatrics 2004; 114 (1): 297-316.

22.- Deis Minsal: Acceso 21 de julio de 2012. http://deis. minsal.cl/vitales/vitales2009/Nacvivos_gestacion_peso_ nacer_2005-2009.htm

23.- Pittaluga E, Díaz V, Mena P, Corvalán S: Curva de Crecimiento Intrauterino para Prematuros entre 23 a 36 semanas de Edad Gestacional. Rev Chil Pediatr 2002; 73 (2): 135-41. 
BARRA L. y cols.

24.- Saigal S, Doyle L: An overview of mortality and sequelae of preterm birth from infancy to adulthood. Lancet 2008; 371: 261-9.

25.- Goldenberg R, Culhane J, Lams J, Romero R: Epidemiology and causes of preterm birth. Lancet 2008; 371 : 75-84.

26.- Volpe J: Neurobiology of Periventricular Leukomalacia in the Premature Infant. Pediatr Res 2001; 50 (5): 55362.

27.- Deis Minsal: Acceso 21 de julio de 2012. http://deis. minsal.cl/vitales/vitales2009/NacVivos_PesoNacer.htm

28.- Hübner ME, Nazer J, Juárez De León G: Estrategias para Mejorar la sobrevida del Prematuro Extremo. Rev Chil de Pediatr 2009; 80 (6): 551-9.

29.- AAP (American Academy of Pediatrics). Engle W, To- mashek K, Wallman C and the Committee on Fetus and Newborn: "Late-Preterm" Infants: A Population at Risk. Pediatrics 2007; 120 (6): 1390-401.

30.- Perlman J: Neurobehavioral Deficits in Premature Graduates of Intensive Care-Potential Medical and Neonatal Environmental Risk Factors. Pediatrics 2001; 108 (6): 1339-48.

31.- Mideplan: El futuro de los niños es siempre hoy. Propuestas del Consejo Asesor Presidencial para la Reforma de las Políticas de Infancia. Consejo Asesor Presidencial Para La Reforma De Las Políticas De Infancia. Chile. 2006.

32.- Mideplan: Sistema de Protección Integral a la Primera Infancia. Chile Crece Contigo. Secretaria Ejecutiva de Protección Social. Ministerio de Planificación, 2006. 